\title{
Dialogue entre expérience et simulation numérique pour un multicristal en alliage à mémoire de forme
}

\author{
Tarek Merzouki $^{1, a}$, Christophe Collard ${ }^{2}$, Nadine Bourgeois $^{3}$, Fodil Meraghni $^{2}$ \\ ET TARAK BEN ZINEB ${ }^{1}$ \\ 1 Laboratoire d'Energétique et de Mécanique Théorique et Appliqué (LEMTA), Nancy-Université, CNRS, 2 rue Jean \\ Lamour 54519 Vandœuvre les Nancy, France \\ 2 Laboratoire de Physique et Mécanique des Matériaux (LPMM), CNRS, Arts et Métiers ParisTech, 4 rue Augustin \\ Fresnel, 57078 Metz, France \\ 3 Laboratoire de Physique et Mécanique des Matériaux (LPMM), CNRS, Université Paul Verlaine de Metz, Ile du Saulcy, \\ 57045 Metz, France
}

Reçu le 20 avril 2009

Résumé - L'objectif de cette étude est de faire dialoguer la simulation numérique et l'expérience pour étudier le comportement en traction d'un multicristal en alliage à mémoire de forme de type CuAlBe. Ce dialogue permet de comparer qualitativement les distributions de transformation martensitique, observée à travers un microscope à grande distance frontale, avec celle obtenue par simulation numérique en utilisant une loi de comportement thermomécanique. Cette loi est basée sur la description de la transformation martensitique à l'échelle du réseau cristallin, elle est implantée dans le code Abaqus ${ }^{\circledR}$. Les conditions aux limites appliquées lors de la modélisation correspondent aux déplacements mesurés sur les sections frontières par une technique de corrélation d'images. Les propriétés géométriques et cristallographiques des différents grains du multicristal sont quantifiées expérimentalement. L'essai de traction est réalisé à température ambiante où l'alliage a un comportement superélastique. Une bonne corrélation entre les observations expérimentales et les résultats numériques est observée.

Mots clés : Corrélation d'images / alliages à mémoire de forme / transformation martensitique / éléments finis / approche micromécanique

\begin{abstract}
Dialog between the experiment and the numerical simulation for a shape memory alloy multicrystal. The aim of this study is to make dialog between the numerical simulation and the experiment in the case of the tensile test behavior for a CuAlBe shape memory alloy multicrystal. This dialog allows to compare qualitatively the distributions of martensitic transformation, observed through a long distance microscope, with obtained by Finite element simulation using a thermomechanical behavior law. The last is based on the description of martensitic transformation on a single crystal scale. It is implemented in the Abaqus ${ }^{\circledR}$ finite element code. The applied boundary conditions for modeling correspond to the measured ones at the edge of the area by a technique of digital image correlation. Geometrical and crystallographic properties of the various grains of the multicrystal are experimentally determined. The tensile test is carried out at room temperature where the alloy has a superelastic behavior. A good agreement between the experimental observation and numerical results is observed.
\end{abstract}

Key words: Image correlation / shape memory alloys / martensitic transformation / finite elements / micromechanical approach

\section{Introduction}

Les alliages à mémoire de forme (AMF) présentent un comportement thermomécanique caractérisé par une

\footnotetext{
a Auteur pour correspondance :

tarek.merzouki@esstin.uhp-nancy.fr
}

transformation de phase. Ce sont des alliages métalliques qui possèdent la propriété remarquable, après avoir été déformés, de pouvoir récupérer leur forme initiale par simple chauffage ou par application d'une contrainte. Généralement, des techniques de transition d'échelle sont utilisées pour la modélisation du comportement des polycristaux en AMF à partir d'une modélisation 


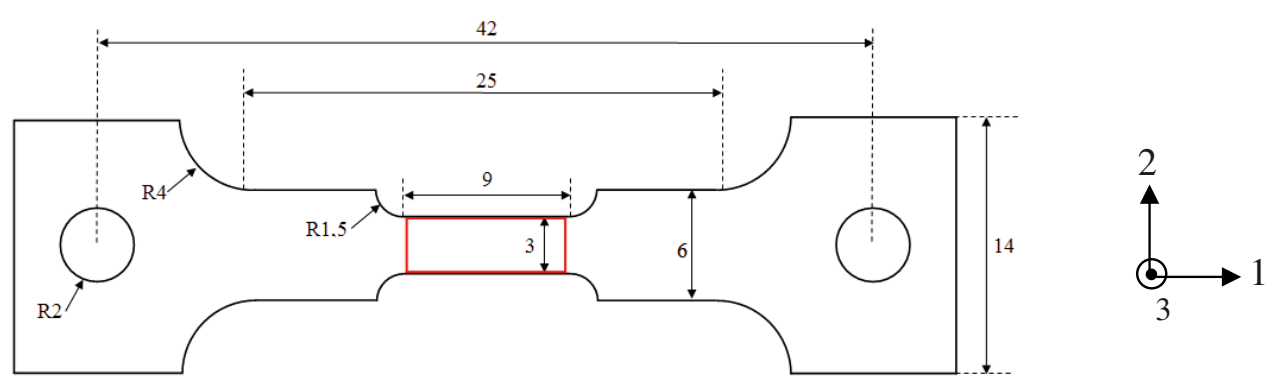

Fig. 1. Géométrie de l'éprouvette, dimensions en mm, (la zone en rouge est la zone d'étude).

micromécanique. Ces approches sont bien adaptées aux polycristaux dont la taille de grains est très faible par rapport à la taille du Volume Elémentaire Représentatif (VER). Dans le cas des multicristaux où la taille du grain est du même ordre de grandeur que la taille du VER, il est nécessaire de prendre en compte les hétérogénéités géométriques et cristallographiques afin d'appréhender leur influence sur le comportement thermomécanique global. Dans cette étude, un modèle éléments finis du multicristal en $\mathrm{CuAlBe}$ a été construit à partir des mesures expérimentales de la forme, de l'orientation cristallographique de chaque grain et du déplacement au niveau des deux sections frontières. Une loi micromécanique monocristalline est adoptée afin de décrire le comportement local de chaque grain. Cette loi est implantée dans le code de calcul par éléments finis Abaqus ${ }^{\circledR}$.

Le champ de déplacement au niveau de la surface libre du multicristal est mesuré par la technique de corrélation d'images numériques. Les images obtenues par une caméra CCD couplée à un microscope sont par la suite traitées par le logiciel Correli-Q4 [1,2] afin d'extraire le champ de déplacement par corrélation d'images, puis le champ de déformation par dérivation numérique. Les résultats numériques issus de la modélisation par éléments finis (déplacements, déformations et fractions de martensite) sont comparés à ceux mesurés ou observés expérimentalement. Ils permettent également d'analyser le comportement du multicristal à l'échelle du grain, et d'effectuer une comparaison qualitative entre la localisation de la transformation martensitique observée expérimentalement, et reproduite au travers de la simulation numérique.

\section{Procédure expérimentale}

Le matériau étudié est un alliage à mémoire de forme à base de cuivre, alliage $\mathrm{CuAlBe}$ fourni par la société « Tréfimétaux », la composition est de $\mathrm{Cu}-11,5 \% \mathrm{Al}$ $0,5 \% \mathrm{Be}$ (\% massique). Ce matériau a été fourni sous forme d'une billette (cylindre) de diamètre $70 \mathrm{~mm}$. Cette billette a été découpée en rondelles de $4 \mathrm{~mm}$ d'épaisseur. Dans une rondelle ont été découpées des bandes de $15 \mathrm{~mm}$ de largeur qui ont été laminées à chaud pour ramener l'épaisseur entre 1 et $2 \mathrm{~mm}$. Plusieurs éprouvettes ont été usinées dans les tôles laminées selon la géométrie présentée dans la figure 1. L'une d'elles, choisie pour cette

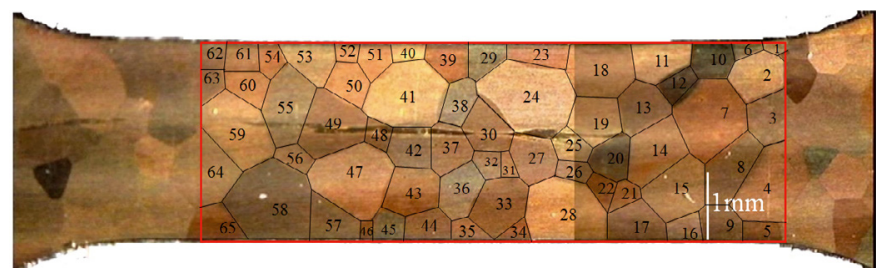

(a)

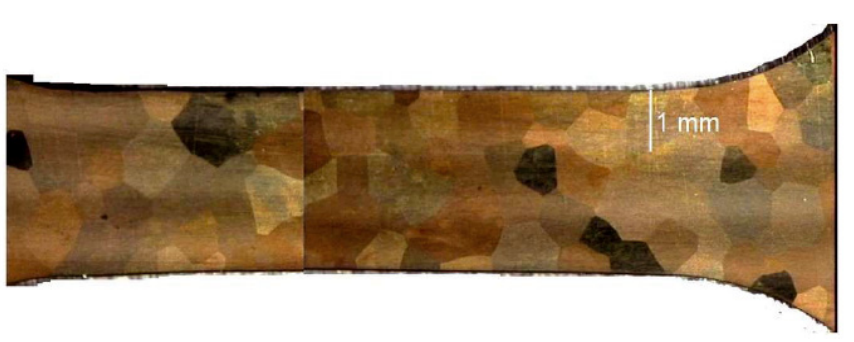

(b)

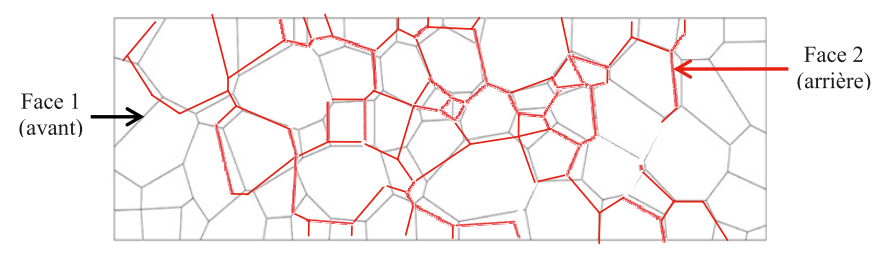

(c)

Fig. 2. Micrographie de l'éprouvette, (a) première face, (b) deuxième face, (c) superposition des faces.

étude a subi un polissage mécanique sur les deux cotés jusqu'à une épaisseur proche de $0,3 \mathrm{~mm}$, suivi d'un polissage électrolytique et d'une attaque colorante qui permet de révéler la microstructure. Les figures $2 \mathrm{a}$ et $2 \mathrm{~b}$ montrent une micrographie des deux faces de l'éprouvette. La zone cadrée en rouge est la zone d'étude. L'échantillon est attaqué seulement après l'analyse EBSD (Electron Back Scatter Diffraction). La taille des grains est millimétrique, très supérieure à l'épaisseur de l'éprouvette. Les grains sont traversants. Les joints de grains dont la géométrie varie assez peu entre les deux faces : figure 2c, ne sont donc pas tout à fait perpendiculaires, ils sont légèrement inclinés. Cependant, dans le cadre de cette étude, l'hypothèse qui est faite de prendre des joints de grains perpendiculaires aux faces de l'éprouvette paraît 
Tableau 1. Orientation cristallographique des grains et le facteur de Schmid dans la direction de chargement.

\begin{tabular}{cccccccccc}
\hline Grain & $\varphi_{1}\left(^{\circ}\right)$ & $\phi\left(^{\circ}\right)$ & $\varphi_{2}\left(^{\circ}\right)$ & $R_{11}$ & Grain & $\left.\varphi_{1}{ }^{\circ}\right)$ & $\phi\left(^{\circ}\right)$ & $\varphi_{2}\left(^{\circ}\right)$ & $R_{11}$ \\
\hline 1 & 242,3 & 22,3 & 54,9 & 0,36 & 34 & 0,1 & 49,9 & 50,4 & 0,39 \\
2 & 341,1 & 37,1 & 17,2 & 0,5 & 35 & 257,4 & 30,3 & 46,6 & 0,25 \\
3 & 355,7 & 31,1 & 84,2 & 0,5 & 36 & 123,2 & 38,6 & 46,2 & 0,44 \\
4 & 99,3 & 27,5 & 76,4 & 0,46 & 37 & 71,8 & 42,5 & 2,2 & 0,32 \\
5 & 59,3 & 29,3 & 31,9 & 0,47 & 38 & 272,2 & 14,8 & 82,5 & 0,49 \\
6 & 294,9 & 41,1 & 52,2 & 0,41 & 39 & 34,8 & 42,3 & 44 & 0,41 \\
7 & 77,5 & 38,2 & 19,8 & 0,41 & 40 & 190,3 & 39,2 & 61,2 & 0,48 \\
8 & 3,7 & 22,1 & 79,2 & 0,5 & 41 & 199,3 & 36,1 & 77,8 & 0,5 \\
9 & 32,9 & 14,4 & 46 & 0,49 & 42 & 160,1 & 24,4 & 66,1 & 0,36 \\
10 & 64,3 & 45,7 & 31 & 0,39 & 43 & 111,7 & 49,9 & 43,4 & 0,34 \\
11 & 228,7 & 30,8 & 50,1 & 0,48 & 44 & 354,8 & 30,3 & 66,2 & 0,46 \\
12 & 307,9 & 27,3 & 46,1 & 0,49 & 45 & 231,2 & 8,4 & 78,7 & 0,37 \\
13 & 344,7 & 21 & 17 & 0,49 & 46 & 47,6 & 43,8 & 48,2 & 0,45 \\
14 & 270,3 & 46,4 & 28,8 & 0,31 & 47 & 344,8 & 37,9 & 35,3 & 0,46 \\
15 & 156,6 & 34 & 7,2 & 0,47 & 48 & 11,7 & 45,7 & 72,8 & 0,48 \\
16 & 250,1 & 32,4 & 6,1 & 0,4 & 49 & 6,3 & 45,2 & 69,5 & 0,49 \\
17 & 336,4 & 18,9 & 49,3 & 0,46 & 50 & 266,7 & 34,9 & 20,2 & 0,38 \\
18 & 29,8 & 31,7 & 80,9 & 0,43 & 51 & 349,9 & 42,3 & 38,6 & 0,44 \\
19 & 12,6 & 38,1 & 22,3 & 0,43 & 52 & 295,6 & 47,3 & 27 & 0,27 \\
20 & 174,8 & 7,2 & 6,9 & 0,48 & 53 & 311,8 & 34,2 & 18,8 & 0,35 \\
21 & 152,2 & 44 & 37,4 & 0,43 & 54 & 34 & 51,9 & 45,8 & 0,37 \\
22 & 152,2 & 44 & 37,4 & 0,43 & 55 & 205,5 & 16,1 & 62,5 & 0,5 \\
23 & 343,9 & 42,2 & 61,4 & 0,36 & 56 & 137,4 & 41,2 & 87,9 & 0,25 \\
24 & 267,4 & 32,8 & 64,3 & 0,29 & 57 & 44,3 & 14,9 & 1,6 & 0,33 \\
25 & 20,9 & 44,1 & 8,8 & 0,43 & 58 & 52 & 15 & 75,7 & 0,37 \\
26 & 351,3 & 17,7 & 24,3 & 0,49 & 59 & 67,3 & 42,4 & 13,3 & 0,36 \\
27 & 256,1 & 40,9 & 19,7 & 0,39 & 60 & 170,9 & 46,6 & 55,5 & 0,38 \\
28 & 7,1 & 38,7 & 24,6 & 0,45 & 61 & 160,9 & 35,9 & 57,6 & 0,35 \\
29 & 208,2 & 45,7 & 55,8 & 0,45 & 62 & 167,2 & 36,7 & 9,2 & 0,5 \\
30 & 117,6 & 22,6 & 73,6 & 0,45 & 63 & 184,1 & 36,1 & 14,8 & 0,49 \\
31 & 167,2 & 46,2 & 52,3 & 0,35 & 64 & 185,5 & 33,5 & 86,1 & 0,49 \\
32 & 26,7 & 33,4 & 27,1 & 0,35 & 65 & 19,4 & 43,9 & 50,3 & 0,43 \\
33 & 6,2 & 32,9 & 51,2 & 0,43 & & & & & \\
\hline & & & & & & & & &
\end{tabular}

raisonnable. La température de début de transformation $M s$ a été mesurée pour le même matériau par DSC [3], elle est comprise entre $-70{ }^{\circ} \mathrm{C}$ et $-85^{\circ} \mathrm{C}$. Le matériau est donc totalement austénitique à la température ambiante. Les essais de traction réalisés dans cette même thèse ont permis d'estimer la contrainte seuil de la transformation à température ambiante : elle se situe entre $160 \mathrm{MPa}$ et $190 \mathrm{MPa}$ [3]. L'orientation cristallographique de chaque grain est déterminée par EBSD. L'indexation des lignes de Kikuchi est effectuée par le système Channel5 (technologie de HKL). Les zones analysées couvrent presque toute la partie calibrée de l'échantillon : trois zones de $2,8 \times 2,8 \mathrm{~mm}^{2}$ sont balayées avec un pas de $1 \mu \mathrm{m}$. La distance de travail est de $15 \mathrm{~mm}$. La tension du faisceau est de $30 \mathrm{kV}$, le courant du faisceau est compris entre 20 et $30 \mathrm{nA}$. Le tableau 1 donne les angles d'Euler déterminés par EBSD en fonction de la position du grain dans le multicristal (Fig. 2a), ainsi que le facteur de Schmid dans la direction de chargement.

\section{Modélisation par éléments finis du comportement d'un multicristal d'AMF}

La loi de comportement utilisée dans cette étude a été développée par Collard et al. [4] en s'appuyant sur le code Simula+ [5]. Elle décrit le comportement thermomécanique du monocristal. Le modèle est basé sur la description de la transformation martensitique à l'échelle du réseau cristallin. Cette loi est implantée dans le code de calcul par éléments finis Abaqus ${ }^{\circledR}$ via la routine UMAT. Toutes les informations complémentaires concernant le modèle et les paramètres régissant le comportement peuvent être obtenues dans la thèse de Merzouki [6]. Certaines caractéristiques du matériau, n'ayant pas pu être identifiées expérimentalement, sont directement reprises de la littérature : tableau 2. La forme de l'échantillon modélisé est présentée dans la figure 3. Seule la partie centrale de l'échantillon est modélisée (cadrée en rouge dans Fig. 1). La zone étudiée comporte 65 grains. Au cours 
Tableau 2. Paramètres matériau, $C_{11}, C_{12}$ et $C_{44}$ sont les constantes élastiques de l'austénite et de la martensite, $\mu$ est le module de cisaillement calculé en fonction des constantes élastiques, $M s$ est la température de début de la transformation martensitique, $A f$ est la température de fin de la transformation inverse (martensite-austénite), $B$ est un coefficient de sensibilité entre la contrainte et la température, les coefficients de compatibilité et d'incompatibilité sont introduits au niveau de la matrice d'interaction entre variantes $[3,7,8]$.

\begin{tabular}{ccccc}
\hline$B\left(\mathrm{MPa} /{ }^{\circ} \mathrm{C}\right)$ & $M s\left({ }^{\circ} \mathrm{C}\right)$ & $A f\left({ }^{\circ} \mathrm{C}\right)$ & $\mathrm{g}$ & \\
\hline 0,2 & -85 & -70 & 0,23 & \\
Coefficient de compatibilité & Coefficient d'incompatibilité & $C_{11}(\mathrm{GPa})$ & $C_{12}(\mathrm{GPa})$ & $C_{44}(\mathrm{GPa})$ \\
$\mu / 350$ & $\mu / 70$ & 142,38 & 95,24 & 124,1 \\
\hline
\end{tabular}

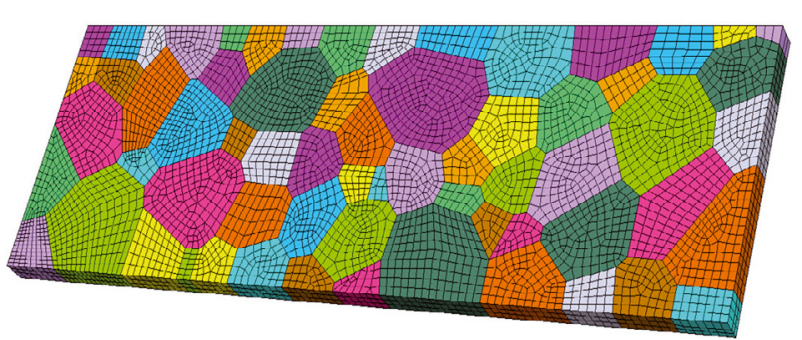

Fig. 3. Maillage adopté (éléments de type C3D8 et C3D6), 12294 éléments.

de la modélisation deux hypothèses ont été adoptées, (i) les joints de grains sont supposés perpendiculaires aux deux surfaces de l'échantillon, (ii) la forme des grains est polygonale. Les dimensions de la partie maillée sont $8,34 \mathrm{~mm}$ de longueur, 2,76 $\mathrm{mm}$ de largeur et $0,27 \mathrm{~mm}$ d'épaisseur. Le maillage de chaque grain est constitué d'éléments cubiques (C3D8) et tétraédriques (C3D6) isoparamétriques à interpolation linéaire. Ils utilisent une méthode d'intégration sélective (Intégration complète de la partie déviatorique et réduite de la partie hydrostatique). Trois éléments sont introduits suivant l'épaisseur bien qu'un seul grain soit considéré suivant cette direction. L'échantillon est maillé avec 12294 éléments. Le chargement est effectué à température constante. Seul le comportement superélastique est exploité. Plusieurs itérations ont été effectuées afin d'aboutir à ce modèle géométrique optimum en terme de rapport précision temps de calcul.

Etant donné que la partie modélisée est située à l'intérieur de la zone maintenue entre les deux mors de la machine de traction, les conditions aux limites doivent être adaptées pour reproduire au mieux le chargement réel. Pour cela, le gradient de déplacement, mesuré par la technique de corrélation d'images, est appliqué en chaque noud des deux sections (gauche et droite) dans les deux directions 1 et 2 (respectivement la direction de chargement et la direction transversale). La figure 4 présente les variations suivant la largeur des deux déplacements mesurés aux limites de la zone maillée lors de l'essai de traction pour un niveau de chargement correspondant à $0,8 \%$ de déformation longitudinale globale. Le déplacement imposé est toujours supposé uniforme dans l'épaisseur. Dans le cas où les positions des nœuds du maillage des deux bords ne correspondent pas aux points de mesures, une interpolation linéaire est effectuée entre les points de mesure afin d'obtenir les déplacements à

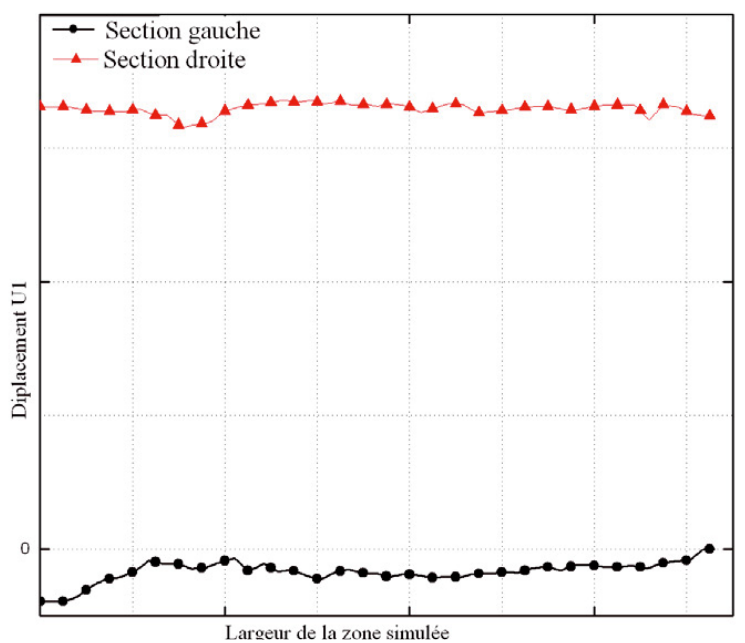

(a)

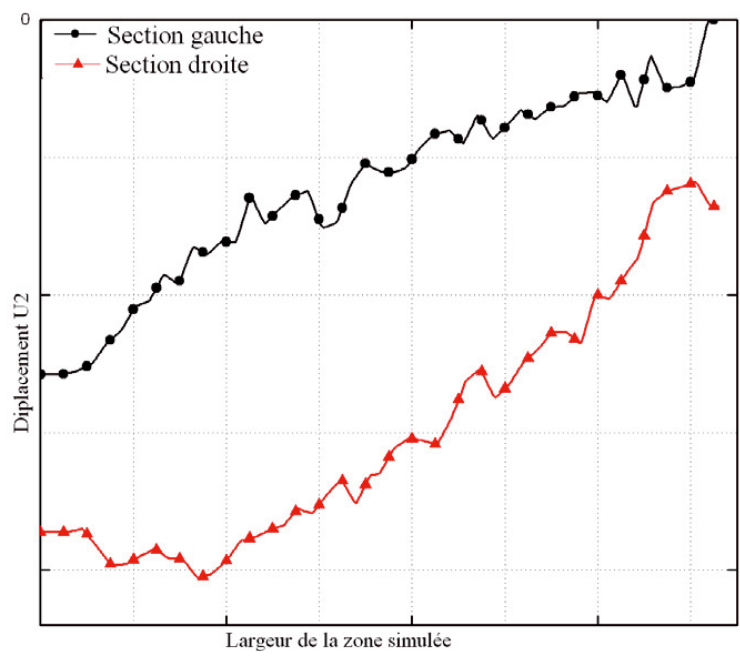

(b)

Fig. 4. Profil des deux déplacements mesurés aux limites de la zone simulées : (a) dans la direction de chargement (U1), (b) dans la direction transversale (U2).

l'emplacement de ces nœuds. Afin d'éviter le déplacement de corps rigide, un seul nœud est fixé dans la direction de l'épaisseur (direction 3). Par l'application du déplacement expérimental en chaque noud des deux bords, il est possible de tenir compte de l'effet des grains voisins sur le comportement de la partie maillée. Enfin, on utilise 


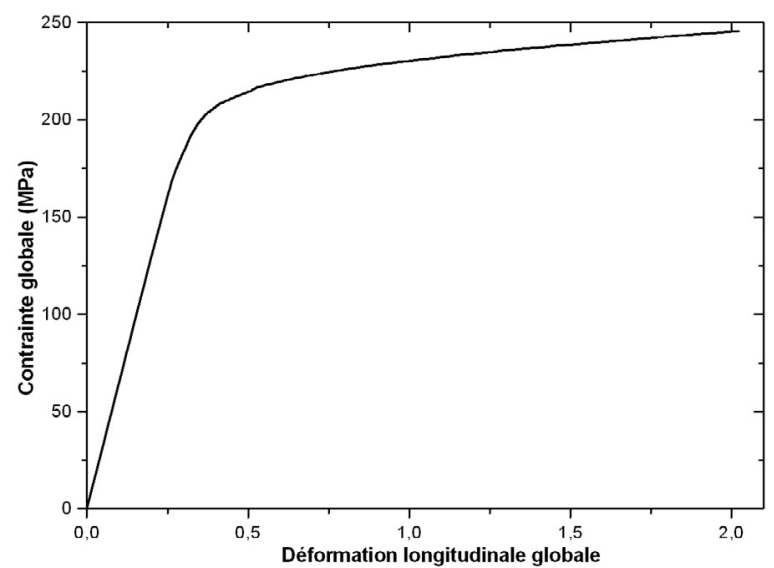

(a)

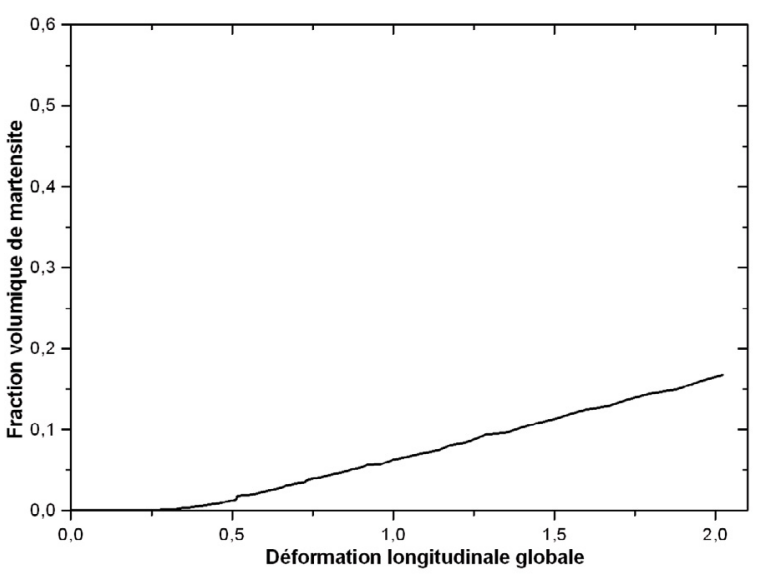

(b)

Fig. 5. Le comportement et la transformation martensitique globale du multicristal, (a) le comportement global, (b) la transformation martensitique globale.

l'hypothèse simplificatrice qui consiste à considérer une variation linéaire dans le temps des conditions aux limites expérimentales.

\section{Analyse des résultats numériques}

La figure 5a présente le comportement global du multicristal, contrainte globale en fonction de la déformation globale. La contrainte globale est calculée à partir de la résultante des réactions aux nœuds d'une section dans la direction de chargement, tandis que la déformation globale est obtenue à partir de la moyenne sur la section des allongements dans la direction de chargement. La figure 5b présente l'évolution de la transformation martensitique globale dans le multicristal en fonction de la déformation globale : l'évolution est quasi linéaire. La transformation apparaît pour un chargement correspondant environ à $0,25 \%$ de déformation globale du multicristal. Jusqu'à ce niveau de chargement,

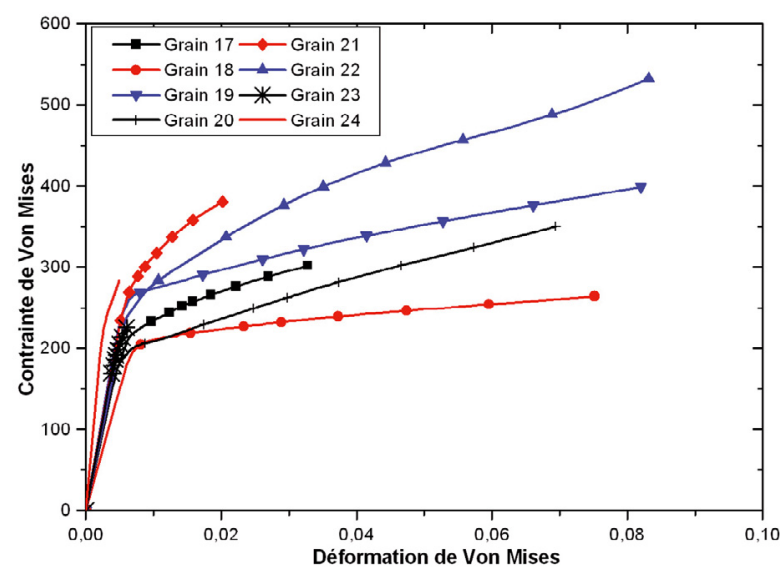

(a)

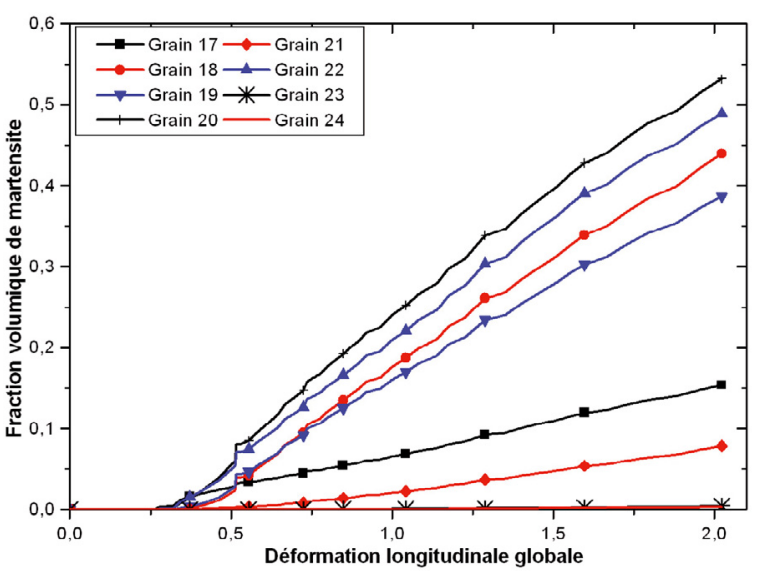

(b)

Fig. 6. Le comportement et la transformation martensitique locale du multicristal, (a) contrainte équivalente de Von Mises en fonction de la déformation équivalente de Von Mises, (b) fraction volumique de martensite par grain en fonction de la déformation globale.

le comportement du multicristal est élastique, cela est confirmé par la courbe du comportement global.

La figure 6a présente le comportement local de quelques grains : la contrainte équivalente en fonction de la déformation équivalente au sens de Von Mises. Ces contraintes et déformations sont moyennées sur tous les éléments appartenant à un grain donné. Pour un chargement correspondant à une déformation globale de $2 \%$, les grains bien orientés par rapport à la direction du chargement présentent un niveau de déformation plus élevé. Par exemple, la valeur locale de déformation est d'environ $8 \%$ pour les grains $18,19,20$ et 22 . Afin de mieux comprendre l'évolution de la transformation dans chaque grain, la figure $6 \mathrm{~b}$ présente l'évolution de la transformation martensitique pour quelques grains représentatifs en fonction de la déformation globale du multicristal. La fraction martensitique présente généralement une valeur plus élevée dans les grains les mieux orientés, par exemple dans les grains 20, 22. Il est à noter que pour 


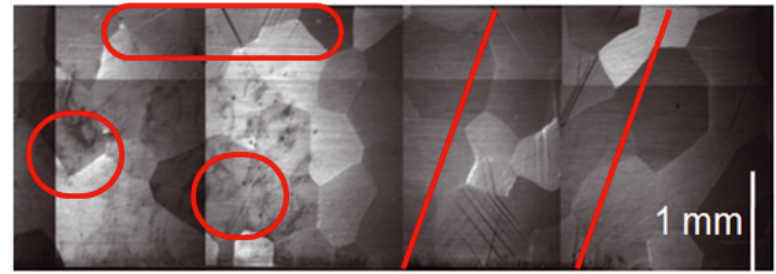

(a)

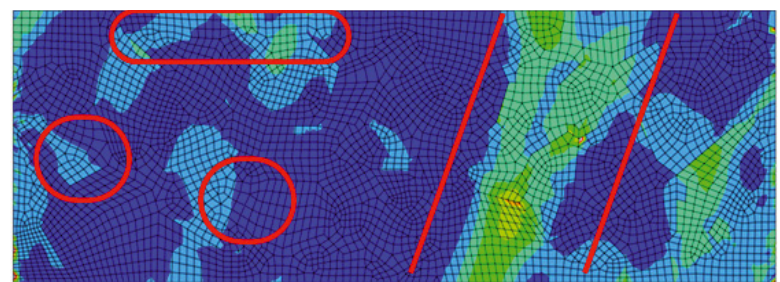

(b)

Fig. 7. Comparaison de la transformation martensitique pour un chargement correspondant à $0,8 \%$ de déformation longitudinale globale (les zones rouges sont les zones de la comparaison), (a) simulation numérique, (b) observation expérimentale.

un chargement correspondant à une déformation globale de $1 \%$, quelques grains ont un taux de transformation martensitique proche de $20 \%$, (grains 20, 22, etc.), tandis que d'autres grains restent dans un état austénitique (grain 23, 24, etc.), avec par conséquent un comportement qui demeure élastique. Enfin, si la fraction volumique de martensite dans le multicristal est d'environ $15 \%$ pour un chargement correspondant à une déformation globale de $2 \%$, elle est proche de $50 \%$ dans les grains 20, 22 .

\section{Comparaison qualitative sur la transformation martensitique}

La figure 7 présente une comparaison entre la transformation martensitique observée expérimentalement à l'aide d'un microscope à grande distance frontale lors de l'essai expérimental pour un chargement correspondant à $0,8 \%$ de déformation longitudinale globale (Fig. 7a), avec celle obtenue par la simulation numérique en appliquant les conditions aux limites mesurées expérimentalement par corrélation d'images (Fig. 7b). Une grande similitude est observée entre les deux résultats. La localisation des bandes de transformation obtenues avec le calcul par EF est semblable à celle observée expérimentalement. Ces résultats montrent l'importance de choisir des conditions aux limites correctes. En effet, ces dernières ont une influence significative sur le développement de la transformation martensitique. Les conditions aux limites expérimentales permettent de respecter les interactions locales entre les grains situés sur les bords de la partie maillée. Elles permettent également de simuler de façon plus réaliste le comportement global du multicristal par l'intermédiaire des interactions entre les grains [10].

Les champs de déplacements et de déformations dans un multicristal à mémoire de forme dépendent

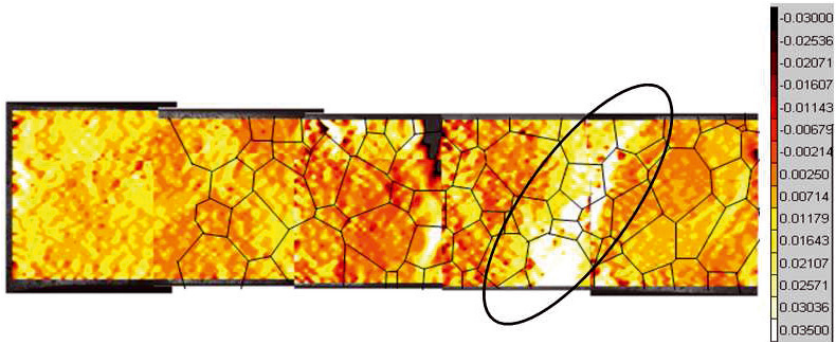

(a) $\varepsilon_{11}$

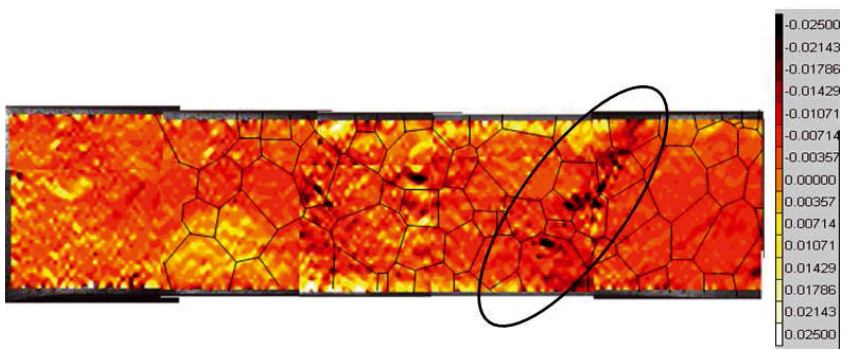

(b) $\varepsilon_{22}$

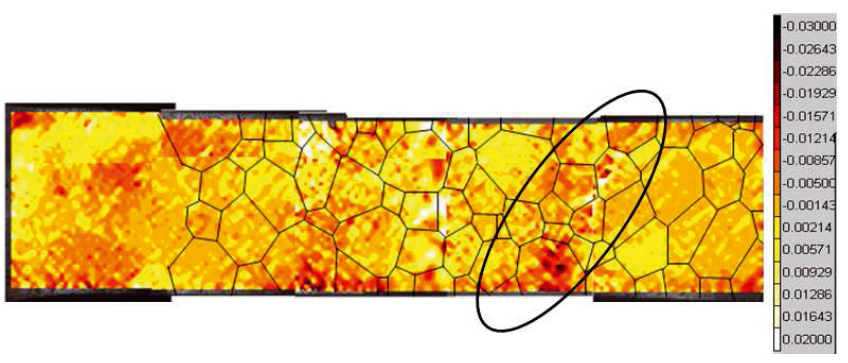

(c) $\varepsilon_{12}$

Fig. 8. Champs de déformations pour un chargement correspondant à $0,8 \%$ de déformation longitudinale globale, suivant la direction de chargement (a), suivant la direction transversale (b), et en cisaillement (c).

fortement des hétérogénéités et des caractéristiques cristallographiques locales (taille de grain, orientation cristallographique, transformation de phase). La figure 8 présente les champs de déformation mesurés par la corrélation d'images sur les différentes zones de l'éprouvette. Elles couvrent la bande où la transformation s'est le plus développée. Les hétérogénéités de déformations élastiques existent, mais sont bien entendu moins importantes que les hétérogénéités atteintes lors de la transformation martensitique. Comme attendu, les déformations sont particulièrement importantes dans les zones transformées [10].

\section{Conclusion}

Dans cette étude nous avons détaillé la confrontation entre calcul numérique et analyse expérimentale pour un multicristal en AMF. Nous avons vu d'une part comment l'expérience nous a fourni certains renseignements indispensables à la simulation du comportement du multicristal et d'autre part, dans quelle mesure l'expérience a 
pu être reproduite par la simulation. Nous nous sommes pour l'instant contentés de comparaisons plutôt qualitatives entre expérience et simulation. Les formes des grains observées en microscopie optique ont été reproduites sur le maillage éléments finis. Un seul grain a été considéré dans l'épaisseur. Les résultats montrent les avantages qu'il y a à appliquer les conditions aux limites expérimentales. Les résultats de la simulation s'approchent alors incontestablement davantage des observations expérimentales. Ceci s'explique par la petite taille de l'échantillon : le volume simulé n'est pas représentatif du comportement moyen du matériau. Les hétérogénéités du comportement ne peuvent pas être négligées, même au niveau de la prise en compte des conditions aux limites, surtout si on veut regarder près des zones où sont appliquées ces conditions aux limites. Cependant, pour une analyse plus fine des résultats obtenus par simulation et leur comparaison avec les mesures de champs, il ne faut pas oublier un certain nombre d'hypothèses. En particulier, n'oublions pas que nous avons utilisé une évolution des conditions aux limites linéaire en fonction du temps et uniforme suivant l'épaisseur. Ces hypothèses, fortement restrictives, ont montré leurs limites en générant un comportement inapproprié avec l'apparition d'un champ de contraintes transversales non négligeables [9].

\section{Références}

[1] S. Roux, F. Hild, Stress intensity factor measurements from digital image correlation : post-processing and integrated approaches, Experimental Mechanics 140 (2006) 141-157

[2] F. Hild, S. Roux, CorreliQ4: A software for "finite element" displacement field measurements by digital image correlation. Internal repart no. 269, LMT-Cachan, 2002
[3] B. Kaouache, S. Berveiller, K. Inal, A. Eberhardt, E. Patoor, Stress analysis of martensitic transformation in $\mathrm{CuAlBe}$ polycrystalline and single crystalline shape memory alloy, Materials Science and Engineering A 378 (2004) 232-237

[4] C. Collard, T. Ben Zineb, E. Patoor, M. Ben Salah, Micromechanical analysis of precipitate effects on shape memory alloys behaviour, Materials Science and Engineering A 481-482 (2008) 366-370

[5] Simula + , est un code scientifique développé en $\mathrm{C}++$ au Laboratoire de Physique et Mécanique de Matériaux, Metz, France, et au Laboratoire de Mathématiques et ses Applications deValenciennes, Valenciennes, France, site Web : http://www.univ-valenciennes.fr/lamav/ Simulat/

[6] T. Merzouki, Identification expérimentale et modélisation micromécanique du comportement d'un multicristal en alliage à mémoire de forme, Thèse de doctorat, Arts et Métiers ParisTech, 2008

[7] D. Entemeyer, E. Patoor, A. Eberhardt, M. Berveiller, Strain rate sensitivity in superelasticity, International Journal of plasticity 16 (2000) 1269-1288

[8] C. Niclaeys, T. Ben Zineb, S.A. Chirani, E. Patoor, Determination of the interaction energy in the martensitic state, International Journal of plasticity 18 (2002) 1619-1647

[9] E. Héripré, M. Dexet, J. Crépin, L. Gélébart, A. Roos, M. Bornert, D. Caldemaison, Coupling between experimental measurements and polycrystal finite element calculations for micromechanical study of metallic materials, International Journal of Plasticity 23 (2007) 1512-1539

[10] T. Merzouki, C. Collard, N. Bourgeois, T. Ben Zineb, F. Meraghni, Coupling between measured kinematic fields and multicrystal SMA finite element calculations, Journal Mechanics of Materials, Soumis (2009) 